\title{
Asian National Adolescent Gambling Surveys: Methodological Issues, Protocols and Advice
}

\author{
Mark D. Griffiths* \\ International Gaming Research Unit, Psychology Division, Department of Social \\ Sciences, Nottingham Trent University, Burton Street, Nottingham, NG1 4BU, \\ United Kingdom \\ *Corresponding author; email: mark.griffiths@ntu.ac.uk
}

\begin{abstract}
This paper provides practical information for researchers in Asia as an aid to develop the methodology for national studies of adolescent gambling and gaming behavior. The paper covers various areas including: (i) optimal study parameters; (ii) age of sample; (iii) time-frame; (iv) number of participants; (v) sampling approach; (vi) questionnaire topics; (vii) paper-based questionnaires versus online questionnaires; (viii) risks and challenges; (ix) practical advice on carrying out a national adolescent gambling survey; ( $\mathrm{x}$ ) obtaining schools' agreement to participate; (xi) setting up the self-completion sessions; (xii) conducting the self-completion sessions; and (xiii) incentives. It is hoped that the somewhat prescriptive advice will aid the gathering of good quality data from adolescent Asian gamblers and help research teams develop robust and strategic research protocols during all stages of the research process.
\end{abstract}

Key words Adolescent Gambling · Problem Gambling · Gambling Methodology $\cdot$ Asian Research

\section{Introduction}

This paper provides practical information for researchers in Asia as an aid to develop the methodology for national studies of adolescent gambling behavior. The areas covered in this paper are based on consultancy and research done by this author with various research bodies (most notably Ipsos MORI and the National Centre For Social Research who were instrumental in developing many of the research protocols outlined here), in addition to the author's own experience of carrying out research in the area of adolescent gambling (e.g., Griffiths, 1995, 2002, 2008).

The paper covers various areas including: (i) optimal study parameters; (ii) age of sample; (iii) time-frame; (iv) number of participants; (v) sampling 
approach; (vi) questionnaire topics; (vii) paper-based questionnaires versus online questionnaires; (viii) risks and challenges; (ix) practical advice on carrying out a national adolescent gambling survey; $(\mathrm{x})$ obtaining schools' agreement to participate; (xi) setting up the self-completion sessions; (xii) conducting the selfcompletion sessions; and (xiii) incentives. Other issues that will be considered include question order, questionnaire development, piloting the questionnaire, cognitive testing, quality control, weighting, informed consent, longitudinal study considerations, interpretation of the data and statistical reliability, profiling young gamblers, peer review, assessment of problem gambling, and further longitudinal study considerations.

\section{Optimal Study Parameters}

Based on the research that this author has carried out over the last 20 years (e.g., Griffiths, 1995; 2002; 2008), it is advised that the optimal way to carry out a national adolescent gambling study (financially, methodologically and practically) is to collect data via the school classroom rather than data collection via parental households. The reasons for this are multiple but in short, access via schools would be substantially cheaper in cost (both time and money) and is likely to achieve a large sample size relatively quickly, with a better response rate. In addition, children are more likely to give honest answers in a setting where there is no chance of their parents or guardians being present.

\section{Age of Sample}

Based on past studies in the area from around the world (e.g., MORI/International Gaming Research Unit, 2006), it is advised that the research team administers a questionnaire to a representative sample of the 12- to 15-year-old population living in the particular Asian country (although some studies worldwide have examined the 11- to 16-year-old age range and this is also acceptable). It is unlikely that a significant proportion of adolescents will be gambling at the age of 10 years and below so 11 years is likely to be an optimal minimum age.

\section{Time-frame}

The time-frame of the study depends upon the exact methodology used and the number of participants surveyed. However, such a survey could be reasonably completed within a 6- to 8-month time period. Table 1 provides a suggested plan with a list of tasks and outputs appropriate to the particular research stage, based on an 8-month time-frame.

\section{Number of Participants}

The number of participants sampled will partly be dependent on the budget available, but for a national population study, a sample size of at least 5,000 participants is recommended. The four national British adolescent gambling surveys have had sample sizes ranging from 8,000 to 11,000 adolescents (Griffiths, 2009) but some Asian countries have a smaller population than Britain. Using a sample size of 5,000 adolescents, given an expected problem gambling prevalence of at least $1 \%$, would mean that the research team would have responses from approximately 
50 problem gamblers on which to do some comparative analysis and statistics. The basic rule of thumb is 'the more the better'; very low response rates would severely affect the validity of any findings (whatever the sample size).

\section{Sampling Approach}

In British surveys of adolescent gambling, a two-stage sampling approach has typically been used (by school, and then by class). Clearly, this author does not know the composition or structure of the typical Asian schooling system but it is assumed that there are broad similarities between Asian and non-Asian school systems. In the first stage, a random sample of schools can be targeted (around 900 schools nationally in Great Britain selected from the national schools' database). These schools can then be invited to participate in the national gambling survey. The school samples can be derived from a wide variety of different types of school. In Great Britain, this can include local educational authority schools, voluntary aided / controlled schools, and foundation schools. Schools that cater for special needs children and sixth-form colleges (that typically teach 16- to 18-year-old

Table 1. Possible research plan and suggested time-frame

\begin{tabular}{|c|c|c|}
\hline Project phase & Timing & Work and outputs \\
\hline $\begin{array}{l}\text { Phase A: } \\
\text { Development }\end{array}$ & Months 1-2 & $\begin{array}{l}\text { - } \text { Methodology reviewed and finalized } \\
\text { - Questionnaire priorities agreed } \\
\text { - } \quad \text { Questionnaire development } \\
\text { - Groups and Steering Groups } \\
\text { - } \quad \text { Gieldwork pilot } \\
\text { - Questionnaire content finalized }\end{array}$ \\
\hline $\begin{array}{l}\text { Phase B: } \\
\text { Fieldwork }\end{array}$ & Months 3-6 & $\begin{array}{l}\text { - } \quad \text { Project specific training sessions conducted } \\
\text { - } \quad \text { Regular monitoring of fieldwork progress } \\
\text { commissioned the research } \\
\text { - } \quad \text { Data checking, editing and cleaning } \\
\text { - Clean, fully derived dataset available }\end{array}$ \\
\hline $\begin{array}{l}\text { Phase C: } \\
\text { Analysis and } \\
\text { archiving }\end{array}$ & Months 7-8 & $\begin{array}{l}\text { - Analysis plan agreed with the agency who } \\
\text { commissioned the research } \\
\text { - Outline report circulated to authors } \\
\text { - } \quad \text { Peer review of initial draft of report (including } \\
\text { response to peer review) } \\
\text { - Continued liaison with the agency who } \\
\text { commissioned the research to review, finalize } \\
\text { and sign off report } \\
\text { - } \text { Key research summary produced } \\
\text { - } \quad \text { Prinal report published } \\
\text { - } \text { stakehtation of results to a variety of } \\
\text { Data deposited with relevant data archiving } \\
\text { organization }\end{array}$ \\
\hline
\end{tabular}


children) are typically excluded from the sampling design. Sampling frames are stratified by Government Office Region and, within each stratum, schools can be selected according to the size of the school register.

In the second-stage sampling procedure, the researchers attempt to survey two class groups (of different age-groups) within each school to provide a sample of 1215 year olds. Typically, the research team selects one class group to be interviewed in each curriculum year, using a random selection procedure. In classes where four or more students are absent during the self-completion session, up to two followup visits are arranged to survey the absent pupils. Using this sampling procedure, around 200 schools would typically participate in the national survey, with 20 to 25 pupils per class completing the paper-based self-completion survey.

\section{Questionnaire Topics and Research Objectives}

A research team may need to decide whether the adolescent gambling survey should emphasize accurate prevalence percentages or if it should look more into the relation between specific behavior and problems. However, it is feasible that both of these objectives can be investigated within the same study. In broad terms, the research objectives for an Asian adolescent gambling survey could be to examine one or more of the following: (i) measure current levels of underage gambling among the Asian country's 12- to 15-year-old age-group, including levels of problem gambling; (ii) compare levels of the Asian country's underage gambling participation among different forms of gambling; (iii) estimate the prevalence of problem gambling among Asian adolescents using validated diagnostic problem gambling criteria; (iv) profile Asian underage gamblers, and those who show a predisposition towards problem gambling behaviors; (v) investigate levels of exposure by Asian adolescents to pseudo-gambling games on the Internet, rates of play of online gambling games, and the participation rates of both online and offline (video) gaming; (vi) provide comparisons (where appropriate) between adolescent problem gambling rates in Asia and elsewhere in the world; and (vii) examine the awareness of gambling advertising among Asian adolescents, and the appeal of gambling and barriers that do, or would, prevent young people gambling.

It may also be useful to examine non-financial forms of gaming in Asian adolescents, such as playing online games like World of Warcraft and gambling-type games on social networking sites, such as Bebo and Facebook, given concerns around these activities (see Griffiths, King, \& Delfabbro, 2009; King, Delfabbro, \& Griffiths, 2010). In essence, a survey could focus on: (i) gambling participation in the past year in general and by gambling form (including non-financial gaming); (ii) gambling participation in the past week in general and by gambling form (including nonfinancial gaming); (iii) internet use and online gambling; (iv) awareness and appeal of gambling advertising; and (v) assessment of problem gambling (using the Diagnostic and Statistical Manual $4^{\text {th }}$ Edition Multiple Response Juvenile [DSMIV-MR-J]; see Tables 2 and 3). There is also a strong association between gambling behavior and other health and lifestyle correlates, such as smoking, drinking and general health status in both adults (Griffiths, Wardle, Orford, Sproston, \& Erens, 2010) and adolescents (Griffiths \& Sutherland, 1998; MORI/International Gaming Research Unit, 2006). Therefore, a research team could also give serious 
consideration to including questions on these additional variables.

\section{Paper-based Questionnaires Versus Online Questionnaires}

There are many advantages of conducting research and collecting data via an online questionnaire. As Wood and Griffiths (2007) point out, researchers do not have to be in the same geographical location as either the participants or fellow research colleagues. Advantages for gaming research outlined by Griffiths and colleagues (Wood, Griffiths, \& Eatough, 2004; Wood \& Griffiths, 2007; King, Delfabbro, \& Griffiths, 2009; Griffiths, 2010) include the fact that the Internet: (i) can be a very useful medium for eliciting rich and detailed data in sensitive areas such as gaming addictions; (ii) has a disinhibiting effect on users and reduces concerns about social desirability. For populations discussing sensitive issues like problem gaming, this may lead to increased levels of honesty and therefore higher validity in the case of self-reports; (iii) provides access to individuals who may not have taken part in the research if it was offline; (iv) has a potentially global pool of participants, therefore researchers are able to study extreme and uncommon behaviors, as well as make cost-effective cross-cultural comparisons; (v) provides access to 'socially unskilled' individuals who may not have taken part in face-to-face situations; (vi) can aid participant recruitment through advertising on lots of different bulletin boards and websites (e.g., gambling chat rooms, gaming forums); (vii) can contain archived material allowing time-based qualitative research to be carried out; and (viii) produces data that in most cases can be automatically transcribed, which suits some particular psychological methodologies (e.g., interpretative phenomenological analysis, discourse analysis, etc.).

However, many of the advantages listed here (e.g., global pool of participants, aid to qualitative research, eliciting detailed information, etc.) do not necessarily aid a quantitative and localized Asian adolescent gambling study. The main disadvantages of online questionnaire use (e.g., potentially biased samples, validity issues) are in many ways no different from those encountered in more traditional offline research approaches. Survey data are necessarily self-report, although the collection of the data online may lower social desirability when responding to questions and therefore increase levels of honesty among participants.

Another issue concerns response rates relating to whether survey questionnaires are completed in an online or offline environment. In the 2007 British Gambling Prevalence Survey (Wardle et al., 2007), all 9,003 participants had the choice to fill out the survey online or offline. However, only $7 \%(N=599)$ completed the survey online. Furthermore, the response rate among participants, who initially told the research team that they would carry out the survey online (52\%), was significantly lower than participants who said they would carry out the survey offline (87\%) (Griffiths, 2010). The reasons for this were thought to be that: (i) some respondents simply used the option to fill out the online questionnaire as a 'soft' refusal, promising the interviewer that they would fill in the questionnaire online, without an intention to do so; (ii) some respondents attempted the online survey but gave up before completion; (iii) offering a choice of completion mode caused respondents to put the task aside and that disrupted the response process; (iv) notices about Internet reporting may have caused respondents with privacy 
concerns to question the confidentiality of the survey. Furthermore, research on web surveys by Wardle and Robinson (2007) concluded that providing participants choice of completion mode did little to help address non-response biases. They argued this was because participants who chose the online option were more likely to be from groups with the greatest propensity to respond to surveys (e.g., managerial and professional groups). Therefore, offering an option may lower response rates and may also contribute to greater non-response bias.

Weighing up the advantages and disadvantages of carrying out a national adolescent gambling study in a classroom situation, a paper-based questionnaire appears to be the best and most practical option, although there is certainly room for debate should a research team wish to follow an online questionnaire option. Much of this may depend upon cultural differences in Internet use. It may be that Asian adolescents are very pro-Internet and would prefer such an option, compared to paper-based methods.

\section{Risks and Challenges}

As with any survey, there are a number of risks and challenges that must be identified, and appropriate strategies developed to help ensure high-quality output is delivered. The key risks and challenges for a research team fall into two broad areas. These are generic risks and challenges associated with conducting national gambling surveys, and project-specific risks and challenges. Generic risks and challenges include: (i) achieving a good response rate (in the face of a declining response to surveys); (ii) minimizing non-response biases; (iii) recruitment of hardto-reach groups; and (iv) completing fieldwork within the time period allocated. Project-specific risks and challenges include: (i) developing a methodology that is accepted by a broad range of stakeholders; (ii) maintaining effective communication with stakeholders; (iii) ensuring comparability with other surveys, whilst developing a survey methodology that is well placed to deliver the data needs of the research team both now and in the future; and (iv) providing internationally comparable data on adolescent gambling.

Developing a methodology that is accepted by a broad range of stakeholders and gaining 'buy-in' to the survey is a complex process. The key to managing this process is a mixture of effective communication, total transparency of methods, clear reasoning for any recommended approach, and both listening to and 'taking on board' concerns and responding to them. This process needs to be carefully managed, to maintain the integrity of the final product and to ensure that the survey fulfils its objectives.

\section{Practical Advice on Carrying Out a National Adolescent Gambling Survey}

The following section provides some practical advice on what a research team needs to know (and do) when carrying out a national prevalence survey on adolescent gambling (although some of these protocols can be used in research more generally).

\section{Setting up a Steering Group}

One of the first things that a research team can do is to set up a Steering Group 
who will help oversee the research process and provide help and advice along the way. The Steering Group should comprise stakeholders, such as key research team personnel, gaming industry personnel (e.g., key people from the Asian gaming industry), government officials with a professional interest in gambling, gambling research experts (national and/or international, etc).

\section{Obtaining schools' agreement to participate}

A pre-notification letter needs to be sent to all sampled schools to inform them that a national gambling survey is taking place and asking them to participate. The letter should explain the background to the research, why the participation of their school in the national survey is important, and should also provide practical details about how the survey process works. Letters should also include a 'reply form' that schools can use to give their written intention to participate (or opt out) of the research. Those who are willing to participate should be asked to provide details of staff contacts and potential dates for the classroom sessions to take place, to help in scheduling. Those schools that are not able to participate should be asked to give the reasons why they are unable to take part. Any schools that do not respond to the initial letter should be telephoned by one of the research team, in order to maximize participation response rates. The initial letter sent should also contain an FAQ (Frequently Asked Questions) sheet which gives teaching staff detailed information about the survey. Anecdotal evidence from the British adolescent gambling surveys (e.g., MORI/International Gaming Research Unit, 2006; Ipsos MORI, 2009) indicates that the FAQ helps to alleviate any concerns that schools may have in relation to data protection issues. In some cases, a letter may also need to be sent to local education authorities asking their permission to approach schools about the research in the first place.

\section{Setting up the self-completion sessions}

Once a school agrees to take part in the survey, the research team needs to make contact with the named member of staff or the head teacher (usually by telephone) and obtain a listing of all classes containing pupils in the classes to be sampled. From this list, the research team can select a class, using a random number grid. In selecting the sample of pupils within each school, the aim is to remove any unnecessary bias, thus ensuring that the sample is as random and representative as possible. To prevent high levels of clustering in the sample (that increases survey design effects and reduces the precision of survey estimates), no more than two classes (each from a different age year) should be selected in each school. Where possible, the research team should ensure that all participating classes are mixed sex (unless in a single sex school) and mixed ability. Typically, this involves completing the surveys during form periods, Citizenship classes, or Personal, Social and Health Education lessons (or the Asian equivalents).

\section{Conducting the self-completion sessions}

Self-completion of questionnaires by pupils should be supervised by researchers experienced in this type of research. Each self-completion session should begin with a short introduction to the pupils to explain the purpose and importance of the 
survey. They should be provided with an opportunity to ask any questions about the survey, and they need to be reassured that all responses will be treated confidentially and anonymously. At this session, they should also be talked through how to complete the survey and any particularly difficult questions should be explained. The teacher should be asked to remain present during the session to help ensure the continued good behavior of the pupils. Typically, pupils have approximately 20 to 25 minutes to complete such questionnaires, with a member of the research team on hand to answer any questions or to give assistance if required. However, this should only concern procedural points, and not to help or discuss what pupils' answers should be, or the wider issues raised by the questionnaire. At the end of the session, each pupil should be provided with an envelope in which to place the completed survey. These envelopes are subsequently collected by the researcher. If four or more pupils are absent from the classroom session, the researcher should arrange to come back and conduct the missing surveys at a later date.

\section{Incentives}

For pupil in-class self-completion surveys, schools need to be guaranteed that all tasks will be carried out by members of the research team, and that there will be little or no additional work for the school. Financial incentives are not normally needed to get the schools to participate in a national adolescent gambling survey. However, in order to thank the schools that agree to help with the research, schools are typically provided with a resource pack (including a CD-ROM and lessonplanning materials) around responsible gambling, to help teachers prepare and deliver lessons on the topic. Pupils are each provided with a pen to complete the questionnaire that they are allowed to keep.

\section{Other Issues for Consideration}

This section briefly outlines other various miscellaneous considerations (in no particular order) that a research team would need to consider when carrying out an Asian national adolescent gambling survey.

\section{Question order}

Question ordering should be considered carefully to ensure that the questionnaire progresses logically through the stages that young people would follow when gambling and gaming.

\section{Questionnaire development}

The draft questionnaire should be developed in conjunction with all the major stakeholders (i.e., the research team and the Steering Group).

\section{Piloting the questionnaire}

With any major piece of work, such as a national adolescent gambling study, rigorous pilot work should be carried out to test how the questionnaire works for the target sample. Typically, a series of cognitive tests should be conducted to test all aspects of the questionnaire (including layout and format, question phrasing, and the comprehensiveness and clarity of response options). Such pilot work can 
be carried out with as few as 15 to 20 adolescents in total. Cognitive testing methods help researchers develop questions that are clear and can be understood by all respondents, as the question developers intended. Such methods draw on cognitive and motivational psychology, and provide a useful framework for understanding the cognitive processes involved in answering survey questions. The emphasis here is on the identification of, and reasons for, problems with questions, rather than quantifying the extent of any problems. In this sense, cognitive methods are akin to qualitative methods, in that large sample sizes are not generally required. Cognitive testing focuses upon the distinct actions that a respondent must perform in order to answer a survey question (i.e., respondents must comprehend the questionnaire and must be able to recall and retrieve the relevant information needed).

\section{Cognitive interviews I}

As mentioned, cognitive interviews are qualitative in nature and can help researchers reduce measurement error by designing questions that respondents understand and are willing and able to answer. For example, a respondent may answer 'yes' to a survey question and show no visible signs of confusion, but may be thinking of something totally different to what the question designer had in mind. This would not be revealed in a standard field pilot test. Specifically, cognitive interviewing techniques focus on four processes: (i) how respondents understand and interpret survey questions; (ii) how they recall information that applies to the question; (iii) the judgments they make as to what information to use when formulating their answer; and (iv) how they respond to the question. Using these techniques enables researchers to examine the question-and-answer process and thus identify problems and suggest solutions and improvements to the final instrument.

\section{Cognitive interviews II}

The two most frequently used cognitive interviewing techniques are 'think aloud' and 'probing'. In the think aloud technique, respondents are asked to say out loud what they are thinking as they go about answering the question. For example, adolescents would be encouraged to articulate what they think the question means, what information they are drawing on to answer the question, what decisions they make about what the question means, or what information is required to answer it and how they provide their (final) answer to the question. In the probing technique, the interviewer will ask specific, usually scripted, questions that provide similar information. Probing can be carried out immediately after the adolescent has provided an answer to each question, or retrospectively after all questions have been asked. Probing can also be used in conjunction with the think aloud technique, to elicit further information from the respondent. One major advantage of using these techniques is that flaws in questions are revealed at an early stage, so that solutions can be sought. In comparison to the potential benefits, the cognitive stage of question testing has a relatively small impact on project timetables and budgets.

\section{Quality control}

Any research team chosen that carries out an Asian national adolescent gambling study must (as a matter of routine) apply very high standards of field management 
and quality control by applying a continuous program of in-field supervision, coupled with telephone and postal cross checking.

\section{Weighting}

In order to ensure that the sample of young people who participate in the research is representative, the data are typically weighted by region and gender.

\section{Response rates}

The success of an Asian adolescent gambling survey will largely depend on minimizing non-response bias as much as possible, so that the research team can be confident that the survey estimates and findings are reliable. One component of this will be to maximize response - the better the response rate, the more representative the survey will be of the adolescent population. High response rates will not necessarily guarantee low bias in cases where respondents and non-respondents are very different, but by having a higher response rate the effects of any difference between respondents and non-respondents on potential bias will be reduced.

\section{Informed consent}

Participation in social surveys, such as participating in a gambling questionnaire, must be with the fully informed consent of the participant. It is therefore necessary to make clear to the young people exactly what is expected of them in the survey, what implications their participation has, and that they can opt out at any stage if they so wish. This is best accomplished by using experienced researchers to explain the survey to potential respondents and to answer any queries they may have.

\section{Longitudinal study considerations}

Although any tracking study (or longitudinal study) should try to keep the same questions over time for comparative purposes, each new phase of the study should include a full-scale review of the questionnaire used in the previous phase. It is likely that many questions on the survey will be identified as key tracking questions, and should therefore be retained in the same (or very similar) format. However, legislative (or other) changes may mean that modifications may need to be undertaken (such as if gambling advertising laws have changed during the course of the study). Other implications about longitudinal studies are highlighted in a separate section later in this paper.

\section{Interpretation of the data and statistical reliability}

When interpreting adolescent gambling survey findings, it is important to remember that the results are only based on a sub-sample of the total population of adolescents in the country, and not on the entire population. Consequently, findings are subject to sampling tolerances, and not all differences between sub-groups will be significant.

\section{Profiling young gamblers}

It would be useful for the research team who carries out this piece of research to include some regression analyses, to gain a deeper understanding of the 
characteristics of young people who gamble, and those who exhibit traits associated with problem gambling.

\section{Peer review}

The research team should be committed to producing the highest quality report possible and as such, they should be committed to the process of peer review. A final report should be reviewed by at least two internationally respected researchers on adolescent gambling. Once peer review comments are received, the research team should address any issues raised and provide a full report of responses and action taken to each peer reviewer.

\section{Assessment of Problem Gambling}

There are now many instruments that measure and diagnose problem gambling. However, very few instruments are specifically designed for use with adolescents. This author recommends using the DSM-IV-MR-J (Fisher, 2000) as this is the mostused questionnaire internationally and would provide comparison rates with studies carried out in places such as Great Britain, Iceland, and other European countries (Meyer, Hayer, \& Griffiths, 2009). Table 2 provides a breakdown of the psychological and behavioral components of the DSM-IV-MR-J Problem Gambling Screen and Table 3 shows how this translates into questions relating to each of the criteria.

Table 2. Psychological/behavioral components of Problem Gambling Screen DSM-IV-MR-J

1. Preoccupied with gambling (e.g., preoccupied with reliving past gambling experiences, handicapping, planning the next venture, or thinking of ways to get money with which to gamble).

2. Needs to gamble with increasing amounts of money to achieve the desired excitement.

3. Restlessness or irritability when attempting to cut down or stop gambling.

4. Gambles as a way of escaping from problems or relieving dysphoric mood (e.g., feelings of helplessness, guilt, anxiety or depression).

5. After losing money gambling, often returns another day in order to get even ("chasing" one's losses).

6. Lies to family members, or others, to conceal the extent of involvement with gambling.

7. Often spends much more money on gambling than intended (loss of control).

8. Commits unsociable or illegal acts, such as spending school fare or dinner money, stealing from the family, or stealing from outside the family in order to finance gambling.

9. Falls out with family, or disrupted schooling (truancy), because of gambling.

Note. DSM-IV-MR-J = Diagnostic and Statistical Manual $4^{\text {th }}$ Edition Multiple Response Juvenile. Source: Ipsos MORI. 


\section{Further Longitudinal Study Considerations}

A large, nationally representative cross-sectional study of Asian adolescent gambling is an ideal starting point for a longitudinal survey or other follow-up study, because it allows for the screening of a large number of individuals from which a relevant sub-sample can be selected. This is a far more robust approach to

Table 3. Problem and social gambler criteria from the DSM-IV-MR-J screen

\begin{tabular}{ll}
\hline DSM-IV criterion & 'During the past 12 months' if any of the following answer \\
criteria are ticked, that qualifies as 1 point
\end{tabular}

1. Preoccupation Have you found yourself thinking about 'Often' gambling or planning to gamble
2. Tolerance Have you needed to gamble with more 'Sometimes' or 'often' and more money to get the amount of excitement you want

\begin{tabular}{|c|c|c|c|}
\hline 3. & Withdrawal & $\begin{array}{l}\text { Have you felt bad or fed up when trying } \\
\text { to cut down on gambling }\end{array}$ & 'Sometimes' or 'often' \\
\hline 4. & $\begin{array}{l}\text { Loss of } \\
\text { control }\end{array}$ & $\begin{array}{l}\text { Have you ever spent much more than } \\
\text { you planned to on gambling }\end{array}$ & 'Sometimes' or 'often' \\
\hline 5. & Escape & $\begin{array}{l}\text { Have you gambled to escape from } \\
\text { problems or when you were feeling bad }\end{array}$ & 'Sometimes' or 'often' \\
\hline 6. & Chasing & $\begin{array}{l}\text { After losing money on gambling have } \\
\text { you returned another day, trying to win } \\
\text { back the money you lost }\end{array}$ & $\begin{array}{l}\text { 'More than half the } \\
\text { time' or 'every time' }\end{array}$ \\
\hline 7. & Lying & $\begin{array}{l}\text { Has your gambling ever led to the } \\
\text { following: telling lies to family / friends } \\
\text { or others }\end{array}$ & $\begin{array}{l}\text { 'Once or twice' } \\
\text { 'sometimes' or 'often' }\end{array}$ \\
\hline
\end{tabular}

8. Illegal acts

Have you ever taken money from any of the following without permission to spend on gambling:

Dinner money or fare money

Money from family

Money from things you've sold

Money from outside the family

Somewhere else
If any one or more of these options are ticked, then qualifies for one point in total
Has your gambling ever led to the following:

(i) arguments with family/friends or others, (ii) telling lies to family/friends or others, (iii) borrowing money from family/friends or others, (iv) missing school
If any of the following are ticked, then qualifies for 1 point in total: 'once or twice', 'sometimes' or 'often'

Note. DSM-IV-MR-J = Diagnostic and Statistical Manual $4^{\text {th }}$ Edition Multiple Response Juvenile. This table indicates how the questions asked in the latest British adolescent gambling survey mapped onto the screen components in Table 1. Source: MORI/International Gaming Research Unit (2006). 
creating a representative sample that includes problem gamblers, than conducting a patron survey, or sampling from treatment facilities. Collecting information from the same individuals over time can enhance our understanding of adolescent gambling and problem gambling in the community over and above what can be learned from the snapshot of behavior obtained in a cross-sectional survey. First, the course of adolescent problem gambling can be delineated. There is emerging evidence suggesting that the course of problem gambling (in both adolescents and adults) is variable - for some individuals, gambling problems are relatively transient, whereas for others, gambling problems are persistent and chronic. This is an important distinction that has largely been overlooked, because until recently, it was assumed that problem gambling was invariably chronic (Meyer et al., 2009). By following individuals who have a range of gambling problems, one can identify those factors leading to the persistence or escalation of problems (versus recovery), as well as identify those factors leading to the initiation of problems.

Second, longitudinal data are essential for establishing the temporal relation that is necessary for inferring a cause-and-effect association. For example, are crimeprone adolescents more likely to become involved in gambling activities, or do gambling activities lead to criminal behavior in some adolescents? Is socioeconomic disadvantage among adolescents a cause or a consequence of problem gambling?

Third, longitudinal data can clarify important findings from cross-sectional research. In Great Britain, in the 1999 national survey (Sproston, Erens, \& Orford, 2000) and to a lesser extent, in the 2007 national survey (Wardle et al., 2007), there was a negative association between age and problem gambling. What was unclear from this cross-sectional data was whether this reflected a developmental trend, or an effect of different rates of problem gambling among individuals from different birth cohorts. The British surveys also showed an interesting pattern of involvement in different gambling activities that suggested a stage-like process that could be confirmed by examining the initiation of new activities over time in a longitudinal study.

Finally, a longitudinal study can potentially provide a sensitive test of the effect of new gambling legislation or policy on adolescent behavior. In effect, this is a 'natural experiment', comparing the same adolescents before and after such a change has been implemented.

In selecting a sub-sample for longitudinal follow-up, it would be important to represent the full range of gambling behavior and consequent pathology. One possibility would be to select all of those individuals who said 'yes' to one or more of the DSM-IV-MR-J screening items in the baseline cross-sectional survey. If a longitudinal study is a serious option, it would be necessary to consider the practicalities, as well as the possible research questions, when setting up the current adolescent gambling survey. The following strategies are therefore recommended:

- Obtaining consent for follow-up (Data Protection Acts around the world usually require that; if a follow-up survey is planned, respondents must give informed consent at the time of first contact. At this stage, this would have to come from the adolescent's parents).

- Obtaining several contact points for respondents (e.g., 'stable' address and telephone number of the adolescent's mother, father and / or guardian). 
- Sending all respondents a 'thank-you' letter for participating in the survey, enclosing a 'change of address' label for respondents to return, free of charge.

- Sending respondents a Christmas card, again enclosing a 'change of address' label for respondents to return, free of charge.

- Setting up a confidential database of respondent contact details, and ensuring that this is kept up to date.

This paper was written as an aid to help research teams in their efforts to carry out an Asian adolescent gambling survey and to provide guidance on what issues need to be considered at all stages of the research process. It is hoped that the somewhat prescriptive advice will aid the gathering of good quality data from adolescent Asian gamblers and help research teams develop robust and strategic research protocols during all stages of the research process.

\section{References}

Fisher, S. (2000). Developing the DSM-IV-MR-J criteria to identify adolescent problem gambling in non-clinical populations. Journal of Gambling Studies, 16, 253-273.

Griffiths, M. D. (1995). Adolescent gambling. London: Routledge.

Griffiths, M. D. (2002). Gambling and gaming addictions in adolescence. Leicester: British Psychological Society/Blackwells.

Griffiths, M. D. (2008). Adolescent gambling in Great Britain. Education Today: Quarterly Journal of the College of Teachers. 58(1), 7-11.

Griffiths, M. D. (2009). Gambling in Great Britain. In G. Meyer, T. Hayer, \& M. D. Griffiths (Eds.), Problem gaming in Europe: Challenges, prevention, and interventions (pp. 103-121). New York: Springer.

Griffiths, M. D. (2010). The use of online methodologies in data collection for gambling and gaming addictions. International Journal of Mental Health and Addiction, 8, 8-20.

Griffiths, M. D., King, D., \& Delfabbro, P. (2009). Adolescent gambling-like experiences: Are they a cause for concern? Education and Health, 27(2), 27-30.

Griffiths, M. D., \& Sutherland, I. (1998). Adolescent gambling and drug use. Journal of Community and Applied Social Psychology, 8, 423-427.

Griffiths, M. D., Wardle, J., Orford, J., Sproston, K., \& Erens, B. (2010). Gambling, alcohol consumption, cigarette smoking and health: Findings from the 2007 British Gambling Prevalence Survey. Addiction Research and Theory, 18, 208-223.

Ipsos MORI. (2009). British survey of children, the National lottery and gambling 2008-09: Report of a quantitative survey. London: National Lottery Commission.

King, D., Delfabbro, P., \& Griffiths, M. D. (2009). The psychological study of video game players: Methodological challenges and practical advice. International Journal of Mental Health and Addiction, 7, 555-562.

King, D. L., Delfabbro, P. H., \& Griffiths, M. D. (2010). The convergence of gambling and digital media: Implications for gambling in young people. Journal of Gambling Studies, $26,175-187$.

Meyer, G., Hayer, T., \& Griffiths, M. D. (2009). Problem gaming in Europe: Challenges, prevention, and interventions. New York: Springer.

MORI/International Gaming Research Unit. (2006). Under 16s and the National Lottery: Final report. London: National Lottery Commission.

Sproston, K., Erens, B., \& Orford, J. (2000). Gambling behaviour in Britain: Results from the British Gambling Prevalence Survey. London: National Centre for Social Research.

Wardle, H., \& Robinson, C. (2007). Who chooses web surveys? Mode choices among Youth Cohort Studies Respondents. Proceedings of the $8^{\text {th }}$ International Conference of the Association 
Mark D. Griffiths

of Survey Computing.

Wardle, H., Sproston, K., Orford, J., Erens, B., Griffiths, M. D., Constantine, R., \& Pigott, S. (2007). The British Gambling Prevalence Survey 2007. London: The Stationery Office.

Wood, R. T. A., \& Griffiths, M. D. (2007). Online data collection from gamblers: Methodological issues. International Journal of Mental Health and Addiction, 5, 151-163.

Wood, R. T. A., Griffiths, M. D., \& Eatough, V. (2004). Online data collection from videogame players: Methodological issues. Cyberpsychology and Behavior, 7, 511-518. 